\title{
Water vapor megamasers in a circumnuclear ionized torus in the Seyfert galaxy Mrk 348
}

\author{
Akihiro Doi* \\ Institute of Space and Astronautical Science, Japan Aerospace Exploration Agency, \\ 3-1-1 Yoshinodai, Chuo-ku, Sagamihara 252-5210, Japan \\ E-mail: akihiro.doidvsop.isas.jaxa.jo

\section{Seiji Kameno} \\ Department of Physics and Astronomy, Graduate School of Science and Engineering, \\ Kagoshima University, 1-21-35 Korimoto, Kagoshima, Kagoshima 890-0065, Japan
}

\section{Satoko Sawada-Satoh}

Mizusawa VLBI Observatory, National Astronomical Observatory of Japan,

Hoshigaoka-cho 2-12, Mizusawa, Oshu, Iwate 023-0861, Japan

\section{Kazuhiro Hada}

INAF, Istituto di Radioastronomia,

Via Gobetti 101, 40129 Bologna, Italy

\section{Takafumi Haga}

Department of Space and Astronautical Science, The Graduate University for Advanced Studies, 3-1-1 Yoshinodai, Chuou-ku, Sagamihara, Kanagawa 252-5210, Japan

\begin{abstract}
We conducted phase-referenced VLBA observations at $1.7-22 \mathrm{GHz}$ for the type-2 Seyfert galaxy Mrk $348(D=62 \mathrm{Mpc})$ which has water vapor megamasers of very broad line width $\left(\sim 100 \mathrm{~km} \mathrm{~s}^{-1}\right)$. The physical origin of the broad-line masers has been debated. We investigated (1) the position-velocity distribution of the masers, (2) a frequency-dependent core shift, and (3) the opacity distribution of free-free absorption in the nuclear region of Mrk 348. In this paper, we report some observational results. The details of the maser velocity field (with infall and rotation), the enclosed mass and the location of the dynamical center, and the structure of the circumnuclear ionized torus will be presented in another paper.
\end{abstract}

11th European VLBI Network Symposium \& Users Meeting,

October 9-12, 2012

Bordeaux, France

\footnotetext{
* Speaker.
} 


\section{Introduction}

Very broad-line $\left(\sim 100 \mathrm{~km} \mathrm{~s}^{-1}\right) \mathrm{H}_{2} \mathrm{O}$ megamasers have been found exclusively in a few active galactic nuclei (AGNs) with strong radio jets, whereas most of the $\mathrm{H}_{2} \mathrm{O}$ megamasers are associated with radio-quiet AGNs and show only a few $\mathrm{km} \mathrm{s}^{-1}$ line width. The physical origin of the broadline masers has been discussed. Peck et al. [四] found that the broad-line masers of Mrk 348 (all of which are redshifted with respect to the systemic velocity) are associated with the weaker side of the jet. Hence, they proposed that these "jet masers" are located in the shocked region between the receding jet and an encroaching molecular cloud. On the other hand, Sawada-Satoh et al. [వ] found that the broad-line masers of NGC 1052 (all of which are also redshifted) are associated with both the approaching and receding jets, which are obscured by free-free absorption, in agreement with the suggestion by Kameno et al. [3] of "infall masers" in a circumnuclear torus.

Mrk 348 is a type-2 Seyfert galaxy at $D=62 \mathrm{Mpc}$ in a nearly face-on viewed spiral galaxy. Falcke et al. [䧃] discovered broad-line $\mathrm{H}_{2} \mathrm{O}$ megamaser emission, which is redshifted by $\sim 130 \mathrm{~km} \mathrm{~s}^{-1}$ from the systemic velocity. Ulvestad et al. [[5] found a sub-relativistic jet speed $(\sim 0.1 c)$ and onesided jet structure from $15 \mathrm{GHz}$ Very Long Baseline Array (VLBA) images. They further proposed the presence of a plasma torus obscuring the receding jet by free-free absorption. On the basis of VLBA images at 1.7, 8, 15, and $22 \mathrm{GHz}$, Peck et al. [四] found that flux variations of masers and continuum emission are correlated, suggesting unsaturated masers, while all maser components arise entirely from a region $<0.25 \mathrm{pc}$ in extent, located toward the north (receding) jet, hence their proposal for the "jet masers" scenario. The jet inclination is unknown.

\section{Observations}

We conducted multi-frequency continuum observations using the VLBA at 1.7-22 GHz. The observations were carried out using the phase-reference pairs Mrk 348-NGC 315 and Mrk 348NGC 266. Additionally, we analyzed archival VLBA imaging data for the $22 \mathrm{GHz}$ water masers.

\section{Results}

The results may be summarized as follows:

1. The VLBA $22 \mathrm{GHz}$ image (Fig. W) revealed that:

(a) all masers are redshifted,

(b) there is an almost symmetric two-sided jet structure, which can be decomposed into six components $\mathrm{C} 1-\mathrm{C} 6$,

(c) $\mathrm{H}_{2} \mathrm{O}$ masers are found on both the northern and southern sides of the jet,

(d) most of the maser components are clustered around the continuum component "CM" (the detailed distribution is not shown in this report), and

(e) a significant velocity gradient is detected in the direction perpendicular to the jet in the maser cluster, whereas the velocity gradient along the jet direction is marginal (not shown). 
2. Multi-frequency phase-referenced astrometry (Fig. (]) at 1.7-22 GHz revealed:

(a) frequency-dependent core positions (“core shift”) for both Mrk 348 and NGC 315 (not shown), and

(b) an asymptotic position for the core at the higher frequencies.

3. Superposition of multi-frequency continuum images at $5-22 \mathrm{GHz}$ (Fig. (B]) revealed:

(a) a spectral index $\alpha>2.5\left(S_{v} \propto v^{\alpha}\right)$ on the north side and jet structure with stronger asymmetry at the lower frequencies, indicating free-free absorption on the north side,

(b) the distribution of free-free opacity along the jet (not shown), and

(c) the positions of the masers and central engine with respect to the distribution of freefree opacity (not shown).
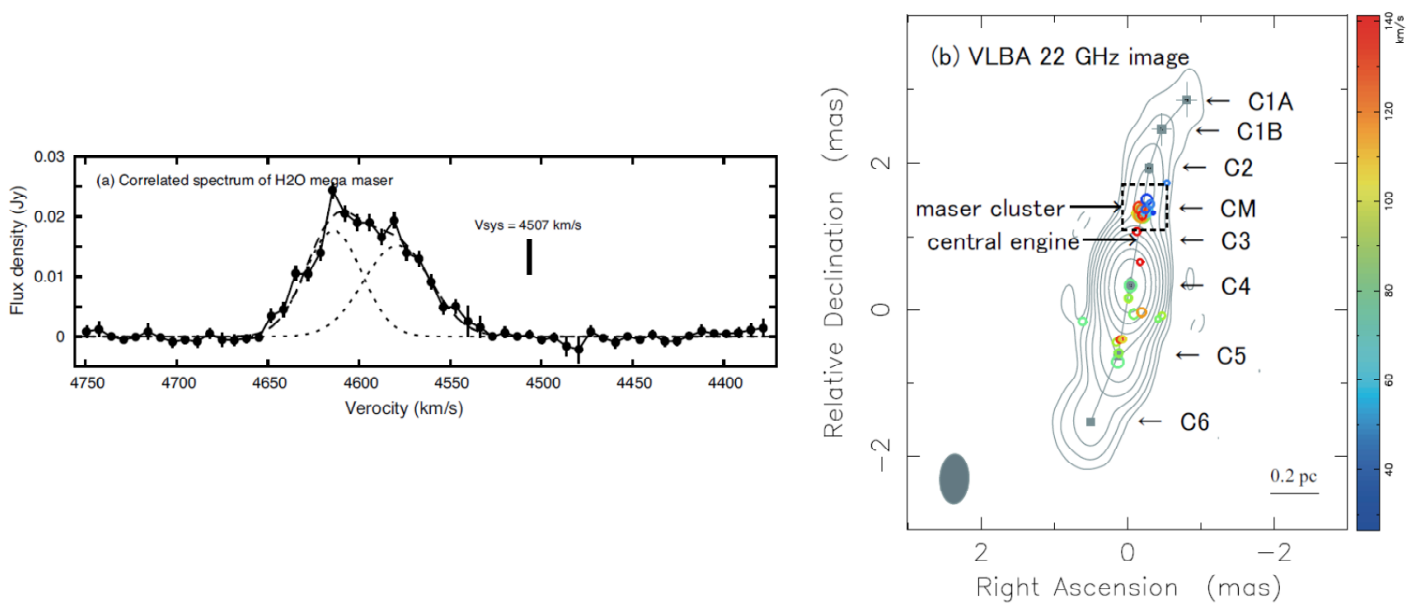

Figure 1: (a) Spectrum of $\mathrm{H}_{2} \mathrm{O}$ maser emission in the $22 \mathrm{GHz}$ VLBA image of Mrk 348. Image noise in linefree channels is $\sim 1.2 \mathrm{mJy}_{\text {beam }}{ }^{-1}$. (b) VLBA image of Mrk 348 at $22 \mathrm{GHz}$. Contours represent the total intensity distribution of continuum emission. Grey symbols represent continuum components identified by model-fitting. Color symbols and their sizes represent the positions of the $\mathrm{H}_{2} \mathrm{O}$ maser components and their flux densities, respectively. The position of the central engine was inferred from core shift measurement.

\section{Conclusion}

Our observational results revealed that Mrk 348 has a circumnuclear obscuring torus containing maser clouds. Additionally, the data should allow us to model the maser velocity field with infall and rotation around the position of a central engine, and to estimate the enclosed mass at the dynamical center. Once final checks for our analyses are made, we will present the details of the observations, analyses, and interpretation elsewhere. 

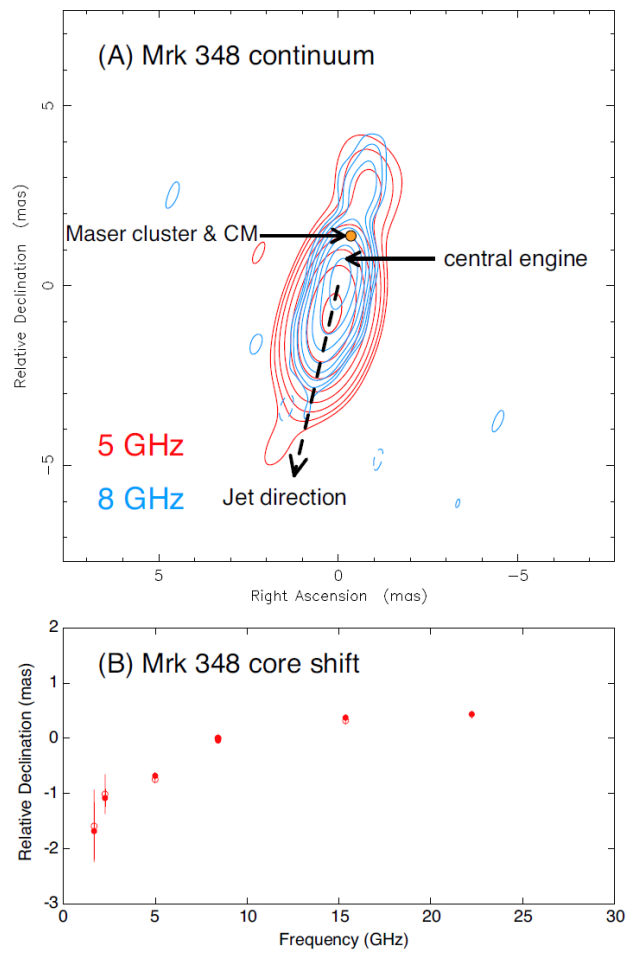

Figure 2: (A) Superposition of VLBA images for Mrk 348 at $5 \mathrm{GHz}$ and $8 \mathrm{GHz}$ after phase-referencing using the reference calibrators NGC 315 and NGC 266. (B) Position of the core as a function of frequency as determined by phase-referencing observations at 1.7-22 GHz. The position of the central engine was inferred from the asymptotic position of the core at the higher frequencies.

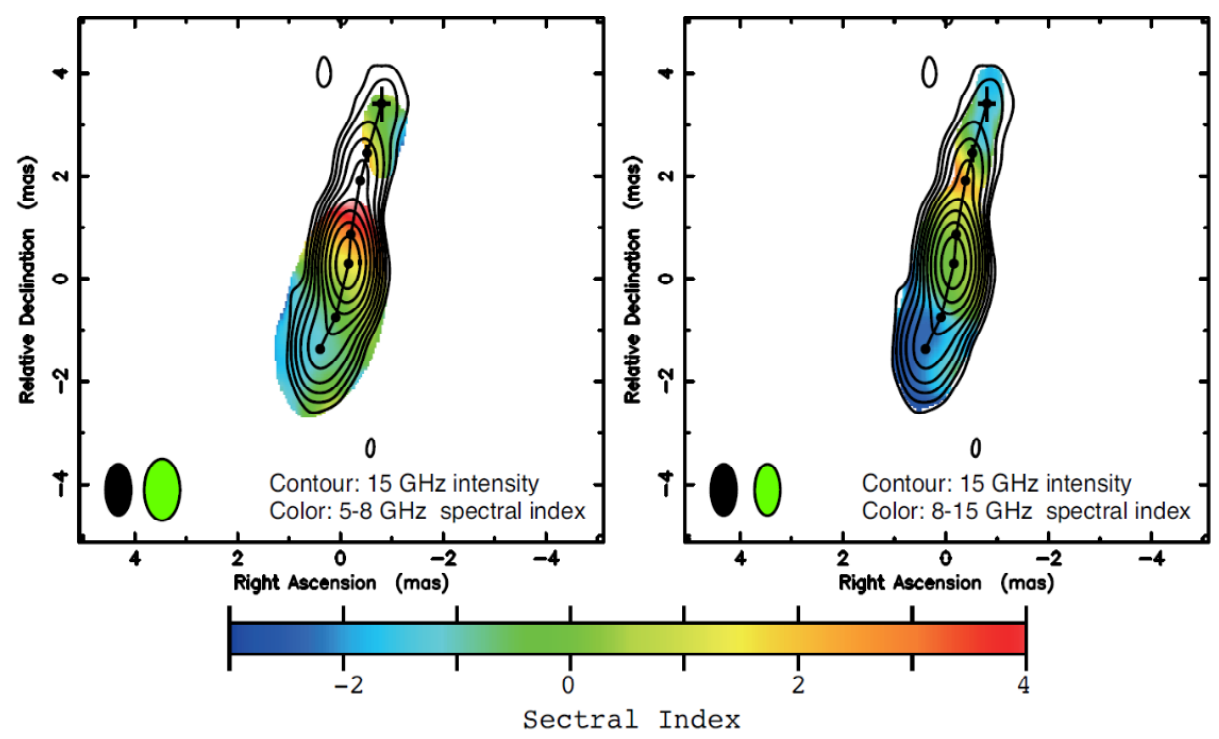

Figure 3: Spectral index maps of Mrk 348 at 5-8 GHz (left panel) and 8-15 GHz (right panel). 


\section{References}

[1] Peck, A. B., Henkel, C., Ulvestad, J. S., et al. 2003, ApJ, 590, 149

[2] Sawada-Satoh, S., Kameno, S., Nakamura, K., Namikawa, D., Shibata, K. M., \& Inoue, M. 2008, ApJ, 680, 191

[3] Kameno, S., Sawada-Satoh, S., Inoue, M., Shen, Z.-Q., \& Wajima, K. 2001, PASJ, 53, 169

[4] Falcke, H., Henkel, C., Peck, A. B., Hagiwara, Y., Almudena Prieto, M., \& Gallimore, J. F. 2000, A\&A, 358, L17

[5] Ulvestad, J. S., Wrobel, J. M., Roy, A. L., Wilson, A. S., Falcke, H., Krichbaum, T. P. 1999, ApJL, 517, L81 